\title{
ORIGINAL ARTICLE \\ The basis of antagonistic pleiotropy in hfq mutations that have opposite effects on fitness at slow and fast growth rates
}

\begin{abstract}
R Maharjan ${ }^{1,2}$, C McKenzie ${ }^{1,2}$, A Yeung ${ }^{1,3}$ and T Ferenci ${ }^{1}$
Mutations beneficial in one environment may cause costs in different environments, resulting in antagonistic pleiotropy. Here, we describe a novel form of antagonistic pleiotropy that operates even within the same environment, where benefits and deleterious effects exhibit themselves at different growth rates. The fitness of $h f q$ mutations in Escherichia coli affecting the RNA chaperone involved in small-RNA regulation is remarkably sensitive to growth rate. E. coli populations evolving in chemostats under nutrient limitation acquired beneficial mutations in $h f q$ during slow growth $\left(0.1 \mathrm{~h}^{-1}\right)$ but not in populations growing sixfold faster. Four identified $h f q$ alleles from parallel populations were beneficial at $0.1 \mathrm{~h}^{-1}$ and deleterious at $0.6 \mathrm{~h}^{-1}$. The $h f q$ mutations were beneficial, deleterious or neutral at an intermediate growth rate $\left(0.5 \mathrm{~h}^{-1}\right)$ and one changed from beneficial to deleterious within a $36 \mathrm{~min}$ difference in doubling time. The benefit of $h f q$ mutations was due to the greater transport of limiting nutrient, which diminished at higher growth rates. The deleterious effects of $h f q$ mutations at $0.6 \mathrm{~h}^{-1}$ were less clear, with decreased viability a contributing factor. The results demonstrate distinct pleiotropy characteristics in the alleles of the same gene, probably because the altered residues in $\mathrm{Hfq}$ affected the regulation of expression of different genes in distinct ways. In addition, these results point to a source of variation in experimental measurement of the selective advantage of a mutation; estimates of fitness need to consider variation in growth rate impacting on the magnitude of the benefit of
\end{abstract} mutations and on their fitness distributions.

Heredity (2013) 110, 10-18; doi:10.1038/hdy.2012.46; published online 21 November 2012

Keywords: Escherichia coli; experimental evolution; fitness variance; growth rate; continuous culture; regulatory mutations

\section{INTRODUCTION}

Fitness increases due to mutations are vital for evolutionary success. The selective advantage of a mutation, $s$, is important because it affects the probability of its fixation in populations (Haldane, 1927). The precise benefit of a beneficial mutation is, however, complicated by several factors, including interactions between mutations (epistasis (Chou et al., 2011; Khan et al., 2011; Ostman et al., 2012)). Mutations in genes that affect more than one trait (pleiotropy (Stearns, 2010)) are also complicated, especially if the pleiotropic mutation negatively affects other cellular properties (antagonistic pleiotropy). With regulatory mutations that exhibit epistasis, pleiotropy or both, the magnitude of $s$ may be subject to factors that lead to its variance in conditions quite close to the selection environment. The range of environments over which $s$ is beneficial is, in general, poorly defined in experimental studies. For mutations exhibiting antagonistic pleiotropy, the question of where in an environmental landscape a mutation switches from beneficial to deleterious is a fundamental one. It is this question we address in the current communication.

Numerous examples of antagonistic pleiotropy have been previously found in laboratory evolution experiments with bacteria, in which fitness gains in the selection environment are due to mutations that affect fitness elsewhere (Cooper and Lenski, 2000; Cooper et al., 2001; MacLean et al., 2004; Ferenci, 2005; Ostrowski et al., 2008). According to Cooper and Lenski (2000), two properties associated with antagonistic pleiotropy are that replicate evolving lines should exhibit parallel decay of functions (those that trade-off with fitness in the selection environment), and that most losses should occur early (when adaptation is fastest). The inverse need not hold, that is, parallel pleiotropic changes are not a necessary consequence of adaptive mutations having antagonistic pleiotropy. Here, we discuss several independently arising $h f q$ mutations in $E$. coli evolving in glucose-limited chemostat cultures that match the Cooper/Lenski predictions and show antagonistic pleiotropy. In contrast to the previously described examples cited above, a novel aspect is that the new $h f q$ mutations switch from beneficial to deleterious even in the same medium environment, but at different growth rates. This unexpected finding prompted us to investigate the mechanism of antagonistic pleiotropy in $h f q$ mutations and also to consider more widely the consequences of growth rate as a factor in $s$ determination.

There is a fundamental reason for considering the relationship of $s$ to growth rate. In an evolving population or in competition experiments, sweeps occur because beneficial or deleterious mutations cause different growth rates in a mutant $\left(\mu_{\mathrm{m}}\right)$ to that found with ancestral bacteria in the same environment $\left(\mu_{\mathrm{w}}\right)$. It matters a great deal how different $\mu_{\mathrm{m}}$ and $\mu_{\mathrm{w}}$ are because physiological studies have demonstrated that growth rate strongly affect patterns of gene expression, ribosome synthesis and metabolic pathways (Tweeddale et al., 1998; Wilson and Nierhaus, 2007; Klumpp et al., 2009; Scott

School of Molecular Bioscience, University of Sydney, Sydney, New South Wales, Australia

Correspondence: Dr T Ferenci, School of Molecular Bioscience, University of Sydney, Sydney, New South Wales 2006, Australia.

E-mail: tom.ferenci@sydney.edu.au

${ }^{2}$ These authors contributed equally to this work.

${ }^{3}$ Current address: School of Civil and Environmental Engineering, University of New South Wales, Sydney, New South Wales, Australia

Received 19 February 2012; revised 10 April 2012; accepted 13 April 2012; published online 21 November 2012 
et al., 2010). Stress responses are increasingly induced at slower growth rates, diverting resources from vegetative fitness functions (Ferenci, 2005, 2007). Hence, a consequence of differences between $\mu_{\mathrm{m}}$ and $\mu_{\mathrm{w}}$ in an evolving population is that an ancestor and mutant inevitably differ in molecular composition in the same environment, not just as a result of a mutation, but also as a consequence of growth rate-determined patterns of gene expression. So how much impact does growth rate context caused by $\mu_{\mathrm{m}}$ have on the estimation of $s$ as a measure of fitness?

Growth-rate affected context can potentially have an impact on fitness and epistatic interactions, especially with the many pleiotropic regulatory mutations important in evolution (Barrier et al., 2001; Philippe et al., 2007). Changes in intracellular context may have profound implications, particularly with regulatory mutations involved in a web of epistatic interactions, (Le Gac and Doebeli, 2010). Evidence for context influence additionally comes from experiments in which applied stresses change the fitness effects of particular mutations (Kishony and Leibler, 2003) and the strong genotype-by-environment interactions in the effects of a global regulatory mutation (King et al., 2006). In most cases, how a mutational benefit interacts with a changing cellular context is not well understood, but a recent study showed fitness of a metal uptake mutation increased with growth rate (Chou et al., 2009).

The variance and magnitude of $s$ are of intense interest in population biology (Orr, 2009), but the interplay between fitness and growth is rarely considered in the experimental assessment of fitness (Chevin, 2011). There is no consistent method of standardizing growth rates during fitness measurements in competition assays and in some studies, fitness is actually measured as a difference in growth rates (MacLean and Buckling, 2009; Lind et al., 2010). In others, $s$ is measured over periods (overnight culture or $24 \mathrm{~h}$ ) in which growth rate is non-constant (for example, (Elena and Lenski, 2003; Perfeito et al., 2007; McDonald et al., 2011)). Here, we directly investigated the fitness of $h f q$ mutants at growth rates in continuous cultures fixed by the chemostat culture method. In a glucose-limited chemostat, bacteria reduce the concentration of limiting nutrient to a level that maintains a constant, suboptimal growth rate. By setting the dilution rate of fresh medium into a chemostat, a range of growth rates can be established. Bacterial growth rate is a saturable function of nutrient level, so residual glucose levels increase with increasing dilution rate. With this system, one can ask not only whether the same mutations are selected at low/high dilution rates (that is, low/high residual resource levels), but also further investigate whether mutations beneficial at one dilution rate are also beneficial at the other dilution rates. Previous studies have shown that some mutations are indeed selected at both $D=0.6 \mathrm{~h}^{-1}$ and $D=0.1 \mathrm{~h}^{-1}$ cultures, but some are not (Notley-McRobb et al., 2003). So here we measured the fitness of pleiotropic mutations under controlled chemostat conditions over a sixfold range of dilution rates $\left(D=0.1-0.6 \mathrm{~h}^{-1}\right)$.

The pleiotropy of $h f q$ mutations is mechanistically interesting because $\mathrm{Hfq}$ is at the center of a wide network of bacterial environmental responses controlled through dozens of small RNAs, Hfq being an RNA chaperone (Tsui et al., 1994; Majdalani et al., 2005). Mutations in $h f q$ cause multiple effects (such as decreased growth yields, altered supercoiling of plasmids in stationary phase, increased cell size, osmosensitivity and increased sensitivity to ultraviolet light (Tsui et al., 1994)), and confer a selective advantage in slow-growing bacteria limited by several nutrients, including glucose, inorganic phosphate and lactate (Conrad et al., 2009; Maharjan et al., 2010; Wang et al., 2010). Evidence described here suggested $h f q$ mutations were less common in fast-growing
$D=0.6 \mathrm{~h}^{-1}$ cultures. The multiplicity of roles of Hfq in imparting a benefit under glucose limitation (Maharjan et al., 2010) also suggested its benefit is sensitive to physiological context. Here, we describe the plasticity of the benefit of several $h f q$ mutations as a function of growth rate in an otherwise constant environment and define how it changes from a beneficial to a deleterious mutation.

\section{MATERIALS AND METHODS}

\section{Bacterial strains and culture conditions}

Bacteria were cultured in L-broth (Miller, 1972) or minimal medium A (MMA) supplemented with either $0.2 \%(\mathrm{w} / \mathrm{v})$ glucose for batch cultures or $0.02 \%(\mathrm{w} / \mathrm{v})$ glucose in chemostats. Media were also supplemented with $1 \mu \mathrm{g} \mathrm{ml}^{-1}$ thiamine and $1 \mathrm{mM} \mathrm{MgSO}_{4}$. For chemostat competition experiments $4 \mu \mathrm{g} \mathrm{ml}^{-1}$ methionine was also added to the medium. Conditions for longterm chemostats are described in Maharjan et al. (2006). All growth was carried out at $37^{\circ} \mathrm{C}$ and the growth rate was controlled by setting the dilution rates of medium supply to the culture vessel according to the standard chemostat practice (Ferenci, 2007).

Strains used in this study are listed in Table 1. Mutant $h f q$ alleles were transferred into ancestral background from the evolved isolates by P1 transduction (Miller, 1972). First, a purA::tet cassette was introduced into the ancestral strain using the protocol described in Yu and Court (1998). The proximity of purA to $h f q$ allowed for $\sim 90 \%$ co-transduction, allowing for selection of purA ${ }^{+}$transductants followed by confirmation of successful $h f q$ transfer by sequencing.

\section{Maximum specific growth rate measurement}

To measure the maximum specific growth rate $\left(\mu_{\max }\right)$, overnight culture of wild-type and $h f q$ mutants grown in $5 \mathrm{ml}$ MMA supplemented with $0.2 \%$ glucose in McCartney bottles were diluted 100-fold in $200 \mu \mathrm{l}$ of the same medium in 96-deep-well plates (clear, polystyrene, flat-bottom from BD, Sydney, NSW, Australia). The plates were incubated in micro-plate reader (BMG Labtech 96, Ortenberg, Germany) at $37^{\circ} \mathrm{C}$ with shaking at 300 r.p.m (double orbital mode). Growth of cultures were monitored by measuring the optical density of cultures at $600 \mathrm{~nm}$ at $15 \mathrm{~min}$ intervals and processed using the BMG Labtech Omega MARS data analysis software (Melbourne, VIC, Australia). The $\mu_{\max }$ of each culture were then calculated from the linear regression of the exponential growth phase after plotting the culture optical density $_{600}$ in logarithmic scale against the time.

\section{Sensitivity to $\alpha$-methyl glucoside}

Sensitivity to $\alpha$-methyl glucoside ( $\alpha-M G)$ was determined by growth on minimal agar plates containing $0.2 \%(\mathrm{w} / \mathrm{v})$ glycerol and $\alpha-\mathrm{MG}$ at a concentration of $0.5 \%(\mathrm{w} / \mathrm{v})$. Strains were cultured overnight in MMA

\section{Table 1 Bacterial strains used in this study}

\begin{tabular}{lll}
\hline Strain & Relevant genotype & Reference \\
\hline BW2952 & F-araD139 D(argF-lac)U169 rspL150 & Ferenci et al. (2009) \\
& $\begin{array}{l}\text { deoCl relA1 thiA ptsF25 flb5301 rbsR } \\
\text { malG: placMu55 (malG::lacZ) }\end{array}$ & \\
BW4001 & Chemostat evolved isolate & Maharjan et al. (2010) \\
$3 \times 17$ & Chemostat evolved isolate & This study \\
$3 \times 27$ & Chemostat evolved isolate & This study \\
BW5155 & BW2952 purA::tet & Wang et al. (2010) \\
BW5035 & BW2952 hfq::amp & This study \\
BW5163 & BW2952 hfqY25D & This study \\
BW5164 & BW2952 hfqL46W & This study \\
BW5165 & BW2952 hfqIS1 & This study \\
BW6034 & BW2952 hfq S60L & This study \\
BW3454 & BW2952 metC::Tn10 & Notley-McRobb and Ferenci, \\
& & (1999) \\
\hline
\end{tabular}


supplemented with $0.2 \%$ glycerol and $2 \mu \mathrm{l}$ of these cultures were spotted onto $\alpha$-MG plates. Plates were incubated overnight at $37^{\circ} \mathrm{C}$ and successful growth indicated resistance to $\alpha-\mathrm{MG}$ (Wang et al., 2010).

\section{Detection of RpoS status}

The level of RpoS was estimated by staining with iodine solution; the intensity of the brown color varies according to the glycogen level in the cell, which in turn is controlled by the RpoS level in the cell (Notley-McRobb et al., 2002).

\section{$\beta$-galactosidase assay}

$\beta$-galactosidase activity of the malG-lacZ fusion present in ancestor and all used strains (Notley and Ferenci, 1995) was determined as described in Miller (1972). For initial characterization, cells were grown in MMA supplemented with $0.2 \%(\mathrm{w} / \mathrm{v})$ glycerol overnight and then subcultured in the same medium until optical density at $600 \mathrm{~nm}$ was $\sim 0.28-0.7$. For characterization of phenotypes in chemostats at different dilution rates, cells were grown in chemostats at the relevant dilution rate for $40 \mathrm{~h}$.

\section{Outer membrane protein analysis}

The outer membrane protein was prepared by disrupting cells in a French pressure cell and separated by SDS-polyacrylamide gel electrophoresis as described previously (Maharjan 2006). Briefly, equal amount of cells $\left(1.6 \times 10^{10}\right.$ cells $)$ from 24 -hour old 80 -ml chemostat cultures were harvested by centrifugation, and the pellet was washed twice with $10 \mathrm{ml}$ of $10 \mathrm{mM}$ HEPES buffer ( $\mathrm{pH} 7.4$ ), resuspended in $10 \mathrm{ml}$ of the same buffer. The cell suspension was then broken by passage three times through a French pressure cell at $6500 \mathrm{lb} \mathrm{in}^{-2}$. The disrupted membrane was pelleted by centrifugation at $35000 \mathrm{~g}$ for $1 \mathrm{~h}$ at $4{ }^{\circ} \mathrm{C}$. The membrane pellet was resuspended in $200 \mu \mathrm{l}$ of sample buffer ( $50 \mathrm{~mm}$ Tris-HCl, $4 \%$ SDS, $16 \%$ glycerol, $0.0048 \%$ bromophenol blue, $4.8 \% \beta$-mercaptoethanol).

The outer membrane samples were boiled for $5 \mathrm{~min}$ at $100^{\circ} \mathrm{C}$ and centrifuged for $10 \mathrm{~min}$ at 14000 r.p.m in an eppendorf microcentrifuge (model 5412). The proteins samples from equal numbers of cells $(10 \mu \mathrm{l})$ were then separated on a $12 \%$ acrylamide gel in the presence of $8 \mathrm{M}$ urea by electrophoresis, and protein bands were stained with $0.04 \%$ Coomassie blue R and destained with $10 \%$ acetic acid.

\section{PCR protocols and mutation analysis}

PCR amplification of a $697 \mathrm{bp}$ region flanking the $h f q$ gene used primers HfqF1 (5'-CGCCAGATGTGGTCTTACCT-3') and HfqR1 (5'-CCTGCTCACCAGCATCATAA- $\left.3^{\prime}\right)$. The reaction profile consisted of 30 cycles of the following in a thermal cycler (Eppendorf Mastercycler): denaturation at $94^{\circ} \mathrm{C}$ for $30 \mathrm{~s}$, annealing at $60^{\circ} \mathrm{C}$ for $30 \mathrm{~s}$ and extension at $72^{\circ} \mathrm{C}$ for $2 \mathrm{~min}$. Product size was confirmed by resolution on a $1 \%(\mathrm{w} / \mathrm{v})$ agarose gel.

PCR products were purified directly using the Wizard PCR preps purification system (Promega Corp., Sydney, NSW, Australia) and sequence analysis of both DNA strands was performed using primers HfqF1 and HfqR1 at the Sydney University Prince Alfred Molecular Analysis Centre (Sydney, Australia). Mutations in $h f q$ were identified by alignment with the known $h f q$ sequence from the National Center for Biotechnology Information database, using BioEdit software (http://www.mbio.ncsu.edu/bioedit/bioedit.html).

\section{Fitness experiments in chemostats and batch cultures}

Fitness comparisons were made against a tetracycline-resistant derivative of BW2952 carrying a metC:: Tn10 insertion in medium supplemented with $4 \mu \mathrm{g} \mathrm{ml}^{-1}$ methionine. This strain was of equal fitness to the ancestor in glucose-limited chemostats, at all dilution rates studied. At least three chemostat competition experiments were conducted as previously described in Maharjan et al. (2010) and Wang et al. (2010), and selection coefficients were based on the equation 11 in Dykhuizen and Hartl (1983) with time measure in hours.

For fitness assay in batch cultures, exponentially growing cultures of reference and test strains in MMA supplemented with $0.2 \%$ glucose and $10 \mu \mathrm{g} \mathrm{ml}^{-1}$ methionine at $37^{\circ} \mathrm{C}$ were mixed equally and diluted 100 -fold in $30 \mathrm{ml}$ of MMA plus $0.2 \%$ glucose and $10 \mu \mathrm{g} \mathrm{ml}^{-1}$ methionine (pre-conditioned at $37^{\circ} \mathrm{C}$ ). The cultures were incubated at $37^{\circ} \mathrm{C}$ with shaking 200 r.p.m.
Samples were taken every hour during the exponential growth phase (at least five time points) and appropriate dilutions were plated on selective L-agar plus tetracycline $\left(15 \mu \mathrm{g} \mathrm{ml}^{-1}\right)$ and non-selective L-agar plates. Selection coefficients of each competing strain were then calculated as in chemostat competitions.

\section{Glucose uptake assay}

For glucose transport assays, $1 \mathrm{ml}$ of culture was extracted from 40 -h-old chemostats and washed in $1 \times$ MMA. Fifty microlitres of this cell suspension

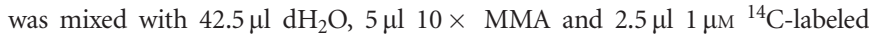
glucose (Amersham, Sydney, NSW, Australia). Samples $(20 \mu \mathrm{l})$ were taken at time points and filtered through pore size $0.45 \mu \mathrm{m}$ filters and washed immediately with $10 \mathrm{ml} 1 \times$ MMA. Uptake rates were determined by measuring scintillation of ${ }^{14} \mathrm{C}$ in the cells in the culture. The optical density at $600 \mathrm{~nm}$ of the remainder of the washed cell suspension was measured in order to estimate the glucose uptake rate in pmol min $10^{-8}$ cells as described in Notley-McRobb and Ferenci (2000)).

\section{Viable cell counts}

Strains were grown in chemostat cultures at the appropriate dilution rate for $40 \mathrm{~h}$ before sampling. The optical density at $600 \mathrm{~nm}$ was measured and the samples were diluted with MMA to a final optical density 600 of 0.1 . The adjusted cell suspension was diluted by a factor of $10^{-5}$ and plated on L-agar plates. Plates were incubated overnight and the resulting colonies were counted to obtain an estimate of the number of colony-forming cells in the chemostat culture.

\section{Estimation of dead cells by propidium iodide staining}

Samples of $\sim 1.5 \mathrm{ml}$ were removed from chemostat cultures after $40 \mathrm{~h}$ and cells were harvested by centrifugation. Cells were then washed once in MMA before being resuspended in $50 \mu \mathrm{l} \mathrm{of} 1 \mu \mathrm{g} \mathrm{ml}^{-1}$ propidium iodide (PI) solution in SSC (sodium citrate solution, $150 \mathrm{~mm} \mathrm{NaCl}$ and $15 \mathrm{~mm}$ sodium citrate) (SigmaAldrich, Sydney, NSW, Australia) and incubated at room temperature for $5 \mathrm{~min}$. Following staining, cells were washed once more in MMA before being spread onto a glass slide. Cells were examined using the BX51 Microscope with Reflection Fluorescence System, Mercury burner (U-RFL-T) and F-view monochrome fluorescence camera and photographed under bright-field and TxRed filters (Olympus, Tokyo, Japan). The numbers of PI-stained cells relative to total cells is a measure of dead cells (Jörgensen and Kurland, 1987) and were counted using ImageJ software (freely accessible from http:// rsb.info.nih.gov/ij/)..

\section{RESULTS}

The biased selection of $\boldsymbol{h} \boldsymbol{f} \boldsymbol{q}$ mutations as a function of dilution rate Analysis of long-term chemostat populations previously discovered evolved clones possessing mutations in $h f q$ in both glucose-limited and Pi-limited chemostats (Maharjan et al., 2010; Wang et al., 2010); these evolution experiments used $D=0.1 \mathrm{~h}^{-1}$ for selection. An easily screenable phenotype of $h f q$ mutations is increased sensitivity to $\alpha$ MG (Maharjan et al., 2010) and this phenotype was used to test the sensitivity of 320 isolates that had evolved in four slow $\left(D=0.1 \mathrm{~h}^{-1}\right)$ and four fast $\left(D=0.6 \mathrm{~h}^{-1}\right)$ dilution rate glucose-limited chemostat populations. After eliminating $\alpha-M G$-sensitive isolates not due to $h f q$ mutations by sequencing the $h f q$ gene in each candidate isolate, four $h f q$ alleles were found among the eight populations. There was a striking bias in the selection of $h f q$ mutants, which were present in independent populations at $D=0.1 \mathrm{~h}^{-1}$ but not at $D=0.6 \mathrm{~h}^{-1}$. One isolated $h f q$ allele was the same Y25D as previously characterized (Maharjan et al., 2010). Other mutations resulted in S60L and L46W substitutions, along with an IS1 insertion $18 \mathrm{bp}$ upstream of the $h f q$ gene (Figure 1a). All isolated $h f q$ mutations reduced function of $\mathrm{Hfq}$ in regulating target genes such as rpoS (Figure 1b, altered glycogen staining with iodine, which is RpoS-dependent (Notley-McRobb et al., 2002)). RpoS is the $\sigma$ factor responsible for general stress 


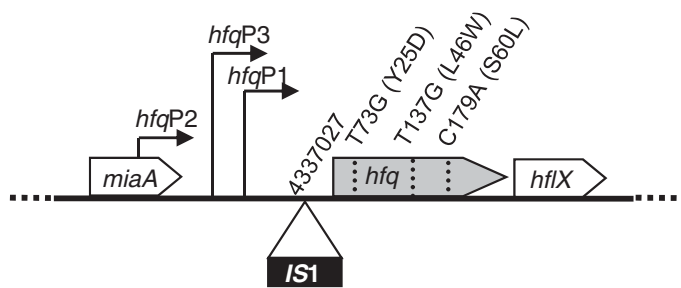

b
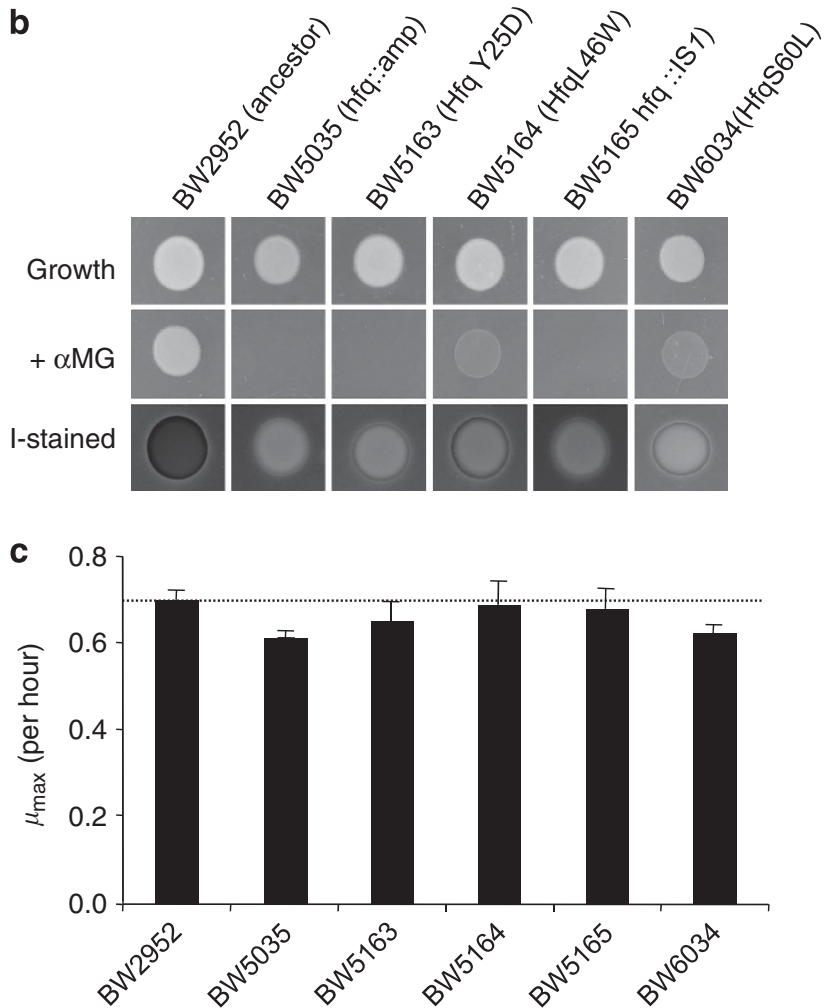

Figure 1 Phenotypic and genotype changes in four $E$. coli clones with different hfq mutations. (a) Schematic diagram of the BW2952 genome segment showing the location of the IS1 insertion in BW5165 and three separate non-synonymous mutations in three different clones. The position of IS 1 insertion in BW5165 was based on the genome position in BW2952 while the positions of point mutations indicated by the vertical dotted lines were based on the $h f q$ gene sequence. Arrows with solid lines indicate positions of the three hfq promoters. (b) The phenotype was characterized in three different ways (from top to bottom): growth on glycerol MMA as a control for the sensitivity assay in the presence of methyl- $\alpha$-glucoside $(\alpha-M G)$; sensitivity to $0.5 \% \alpha-M G$ in a glycerol plate; staining with iodine for RpoS status is shown in the bottom row. (c) Maximum growth rates were measured for four hfq mutants and two controls, the ancestral strain and its $h f q$ knockout derivative. The rate was calculated from the exponential growth phase of cultures growing aerobically in MMA containing $0.2 \%$ glucose at $37^{\circ} \mathrm{C}$. At least ten independent time points were used to estimate the growth rate. The Y-axis error bars were calculated from at least six independent experiments.

resistance, so Hfq indirectly affects many phenotypes associated with stress resistance. As expected, the mutations also affected $p t s G$ function (Figure 1b) as shown by increased sensitivity to $\alpha$-MG (Maharjan et al., 2010). Both rpoS and $p t s G$ are regulated by Hfq in a small-RNA-dependent manner (Gottesman, 2004). The various $h f q$ mutations had broadly the same phenotypic effects, although the magnitude of these effects was dependent on the nature of the mutation (Figure 1b).
The fitness of $h f q$ mutations as a function of dilution rate In view of the pattern of selection found for $h f q$ mutants, we next investigated whether the selective advantage of the $h f q$ mutations was growth rate-dependent. The $h f q$ alleles were individually transferred by $\mathrm{P} 1$ transduction into the ancestor. The resulting strains, as well as an $h f q:: a m p$ knockout strain, were competed against the ancestral strain in glucose-limited chemostats over a sixfold range of dilution rates. In this study, growth rate is measured as $\mu$, equivalent to $r$ in Malthusian growth models, and called the specific growth rate in bacterial growth equations. When growing in chemostats, $\mu$ is set by the dilution rate $D$, and $D=\mu$ at pseudo-steady state (Ferenci, 2007), so different growth rates were experimentally established by setting $D$ between $0.1-0.6 \mathrm{~h}^{-1}$, equivalent to doubling times between $69 \mathrm{~min}$ and nearly $7 \mathrm{~h}$.

The different evolved $h f q$ mutations all provided positive fitness at $D=0.1 \mathrm{~h}^{-1}$ (Figure 2), explaining the selection of the mutants in evolving $0.1 \mathrm{~h}^{-1}$ populations. The constructed $h f q$ knockout strain, however, was not fitter at any dilution rate. This suggests that residual Hfq activity is needed for some cellular functions; complete loss of function mutations were indeed not selected in chemostats.

Interestingly, the selection coefficient of each of the $h f q$ mutants was found to vary markedly with changing dilution rate (Figure 2). Most strikingly, the measured selection coefficients all changed sign at some point as dilution rate increased, although the position and speed at which this occurred differed between alleles, indicative of antagonistic pleiotropy. Particularly the point mutations Y25D and S60L showed a drastic change in fitness, sharply decreasing as dilution rate approached $D=0.6 \mathrm{~h}^{-1}$. In competitions at the maximal growth rate $\left(\mu_{\max }\right)$ in batch culture (batch culture because chemostats do not allow competition at $\mu_{\max }$, as cells are washed out), relatively small differences between mutants and wild-type were found.

Another interesting result was that the range of fitness effects with the set of evolved alleles differed with dilution rate. As indicated by the s.d's in Figure 3a at each growth rate, the five different alleles showed the most divergent fitness effects at low dilution rates but converged and were more similar at fast dilution rate and at the $\mu_{\max }$. As further shown in Figure 3b, particular alleles were more prone than others to have a wide range of $s$ values at different growth rates. So the net fitness effects of $h f q$ mutations are growth-rate affected, although the small sample of mutations in a single gene limits conclusions for fitness distributions in general.

The molecular basis of fitness as a function of dilution rate We attempted to define both the beneficial and deleterious effects of $h f q$ mutations that varied with growth rate. Two $h f q$ alleles that showed different fitness profiles ( $h f q \mathrm{Y} 25 \mathrm{D}$ and the $h f q$ knockout strain) were chosen to observe variation in phenotypic effects across three dilution rates $\left(D=0.1,0.4\right.$ and $\left.0.6 \mathrm{~h}^{-1}\right)$.

First tested was glucose uptake rate, which is the main fitness determinant in glucose-limited chemostats (Ferenci, 2007); this was measured by following the uptake of ${ }^{14} \mathrm{C}$-glucose by the $h f q$ mutants and the ancestor (Figure 4a). At the beneficial dilution rate of $D=0.1 \mathrm{~h}^{-1}$, both $h f q$ mutants show a significantly higher glucose uptake rate than the ancestor (two-tailed $t$-test $P=0.008$ ). A major contributor to this difference is the elevated transport through PtsG in $h f q$ mutants (Maharjan et al., 2010). The transport difference is smaller but still positive at $D=0.4 \mathrm{~h}^{-1}$. At $D=0.6 \mathrm{~h}^{-1}$, the glucose uptake rate of the ancestor and the $h f q \mathrm{Y} 25 \mathrm{D}$ mutant is not significantly different (two-tailed $t$-test $P=0.77$ ), however, the $h f q$ knockout mutant shows a lower glucose uptake rate than either (twotailed $t$-test $P<0.005$ for both strains). The convergence of uptake 


\section{a}

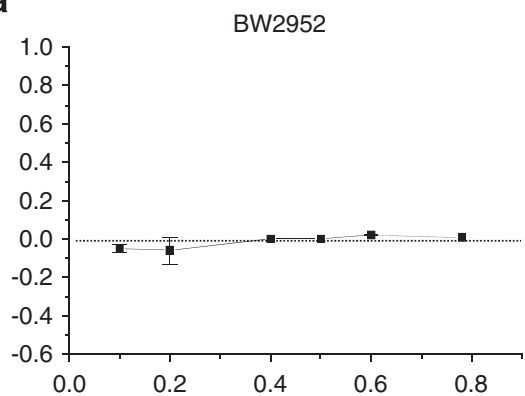

C
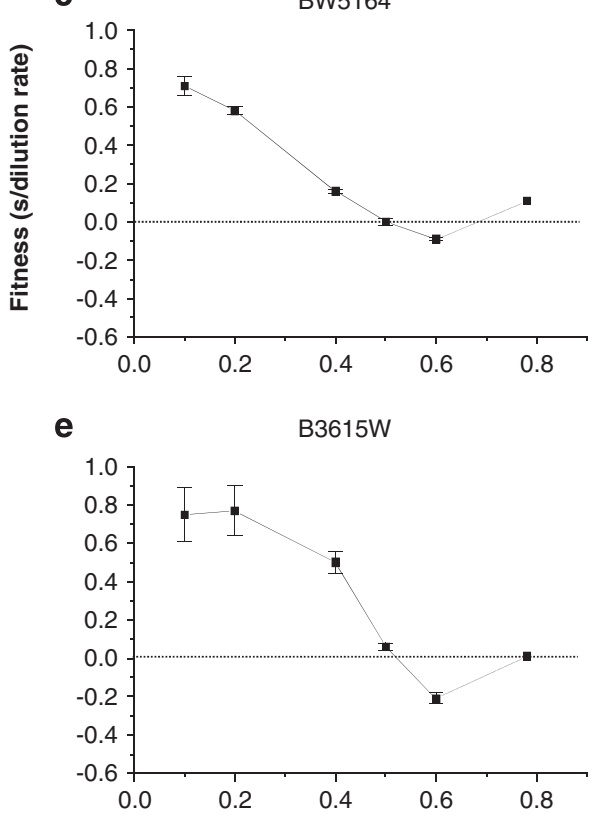

b

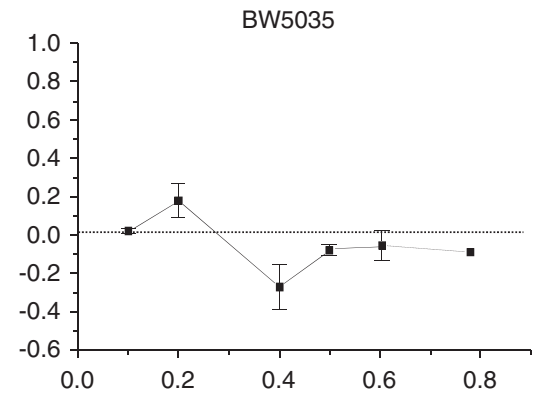

d

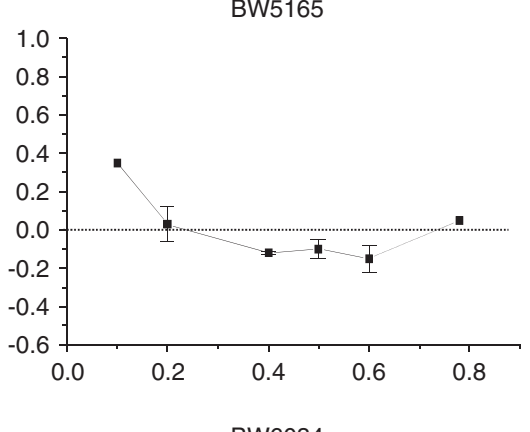

f

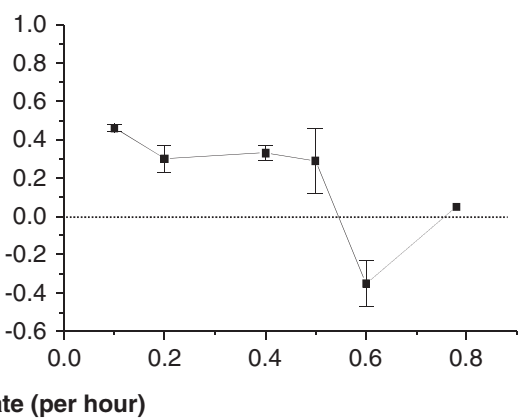

Figure 2 Effects of hfq mutations on competitive fitness and maximum growth rate. Competitive fitness was measured for strains bearing the shown mutant hfq alleles in glucose-limited chemostats (Ferenci, 2007). Competitions were against a reference strain BW3454, which is the ancestral strain but containing a metC::Tn10 countable marker. This reference strain was neutral in fitness when competed against the ancestor as shown in (a). Competing strains were mixed to an $\sim 50: 50$ ratio after $16 \mathrm{~h}$ individual acclimatization at the appropriate dilution rate. In addition to chemostat experiments, the same strains were also competed in glucose-excess batch culture at the $\mu_{\max }$, which cannot be achieved in chemostats; the results in batch culture are plotted joined by dotted lines. Changes in population proportions were used to calculate selection coefficients (Dykhuizen and Hartl, 1983). To eliminate the effect of different generation times, the data is presented as $s / \mu$ (Chevin, 2011). The mean and s.d.'s were obtained from three replicate experiments. The different $h f q$ mutants are shown independently in (b) $h f q$ ::amp knockout, (c) L46W mutant (d) IS 1 insertion in $h f q$ promoter, (e) Y25D mutant and (f) S60L mutant. Dashed lines between $D=0.6$ and $0.8 \mathrm{~h}^{-1}$ indicate the competitions performed at $\mu_{\max }$ or batch cultures.

rates and the major benefit being exhibited at slow growth rate can be explained to be due to two previously determined factors in patterns of gene regulation. In addition to the $p t s G$ effect, Hfq contributes to the high RpoS levels in ancestral bacteria at $D=0.1 \mathrm{~h}^{-1}$, and reduced Hfq function removes the negative effect of RpoS on transport (Maharjan et al., 2010). RpoS is a lesser negative influence at the higher growth rate because it is not so highly expressed (Notley and Ferenci, 1996). In addition, wild type cells naturally optimize their transport at $D=0.6 \mathrm{~h}^{-1}$ by inducing an alternative $\mathrm{Mgl}$ transport system (Ferenci, 1996).

Consistent with the above explanation, the differential effect of dilution rate on the expression of glucose transporters is illustrated by the level of a malG::lac $Z$ reporter gene fusion. The mal genes are known to regulate expression of the outer membrane glycoporin lamB (Notley and Ferenci, 1995), which is involved in glucose uptake at limiting glucose concentrations (Death et al., 1993). The malG::lacZ fusion is also indirectly regulated by $h f q$ (Maharjan et al., 2010) $\beta$-galactosidase assays were carried out on chemostat cultures of the ancestor and both $h f q$ mutants at the three dilution rates tested above (Figure $4 \mathrm{~b}$ ). At $D=0.1 \mathrm{~h}^{-1}, \beta$-galactosidase activity was significantly higher in both $h f q$ mutants (two-tailed $t$-test $P<0.002$ for both strains) than in the ancestor strain, which shows very low levels of expression under these conditions. At this dilution rate, $\beta$-galactosidase activity in the $\Delta h f q$ mutant is also significantly higher than the hfqY25D mutant (two-tailed $t$-test $P=0.001$ ). At $D=0.4 \mathrm{~h}^{-1}$, malG expression is significantly higher for both the ancestor and the $h f q$ Y25D mutant than at 0.1 (two-tailed $t$-test $P<0.002, P=0.004$, respectively), while levels of expression in the $h f q$ knockout mutant converge with those for $h f q \mathrm{Y} 25 \mathrm{D}$ (two-tailed $t$-test $P=>0.05$ ). At $D=0.6 \mathrm{~h}^{-1}$ however, there is no significant difference between either of the $h f q$ mutants and the ancestor (two-tailed $t$-test $P>0.05$ for both strains). The high expression of transporters like LamB protein 

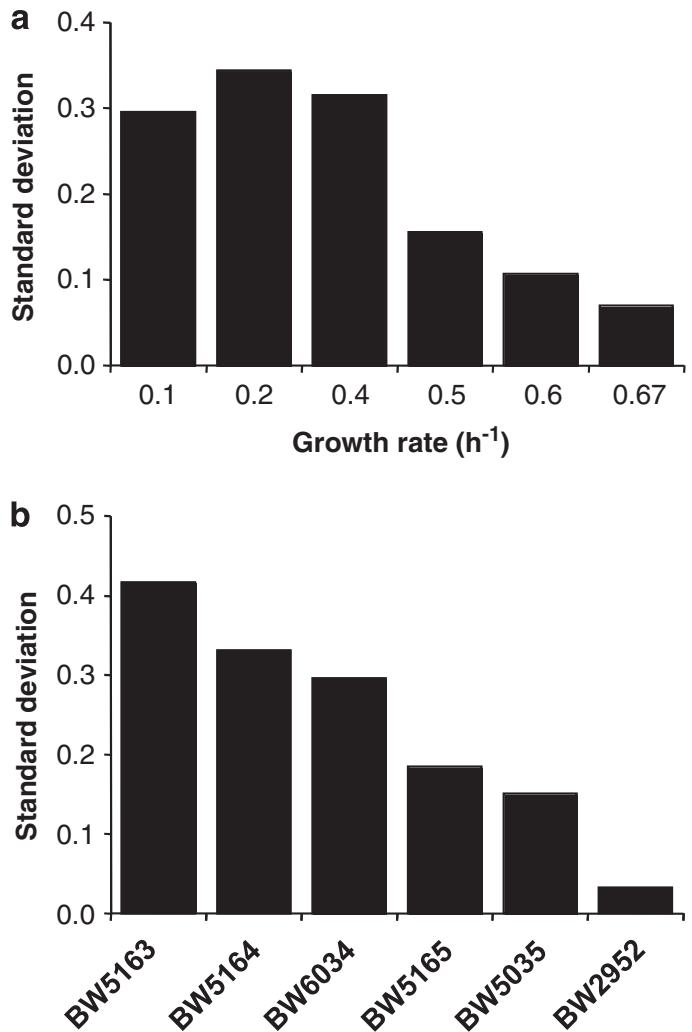

Figure 3 The variance of fitness effects in hfq mutants at different growth rates. In (a), the fitness estimates from Figure 2 were used to calculate s.d.'s at each given dilution rate, using the values from the four independently evolved isolates. In (b) the s.d. was measured for each allele, with the fitness values included from all growth rates.

relative to other major outer membrane proteins at $D=0.6 \mathrm{~h}^{-1}$ is also shown in Figure 4c, comparing equal numbers of extracted cells. At this dilution rate, the $h f q$ mutations have little influence on the LamB level. In contrast, at $D=0.1 \mathrm{~h}^{-1}$, the ancestor has repressed LamB and the $h f q$ mutations elevate LamB levels to that seen at $D=0.6 \mathrm{~h}^{-1}$. Overall, the difference in transport benefit due to the $h f q$ mutations is highly dilution rate-dependent because the cellular context is markedly different between $D=0.1$ and $0.6 \mathrm{~h}^{-1}$, as shown by all the results in Figure 4.

The deleterious effects of the $h f q$ mutations were also investigated. Previous studies have shown that a mutation in $h f q$ causes cell division errors (Tsui et al., 1994) and reduced cell viability (Večerek et al., 2008). To understand if the deleterious fitness effect of $h f q$ mutations at $D=0.6 \mathrm{~h}^{-1}$ was due to increased cell death in the population, we measured the proportion of live and dead cells after staining with PI across the three dilutions rates $(D=0.1,0.4$ and $\left.0.6 \mathrm{~h}^{-1}\right)$. As shown in Figure 5, dead cells in populations of both Y25D and $\Delta h f q$ mutants were more common, compared with ancestor at $D=0.6 \mathrm{~h}^{-1}$. Also at $D=0.6 \mathrm{~h}^{-1}$, we found the proportion of PI staining was around 11 and 19\% compared with that of 5 and $6 \%$ at $D=0.1 \mathrm{~h}^{-1}$ for $\mathrm{Y} 25 \mathrm{D}$ and the $h f q$ knockout strain, respectively, (two-tailed $t$-test $P=0.019,0.023$, respectively). On the other hand, the PI assays indicated that the viability of the ancestral strain remained unchanged with all the three dilution rates (Figure 5) so the dilution rate-dependent decrease in viability could contribute to the reduced fitness of the $h f q$ mutants at $D=0.6 \mathrm{~h}^{-1}$. However, the viability drop between $D=0.4$ and $0.6 \mathrm{~h}^{-1}$ was less drastic than the a
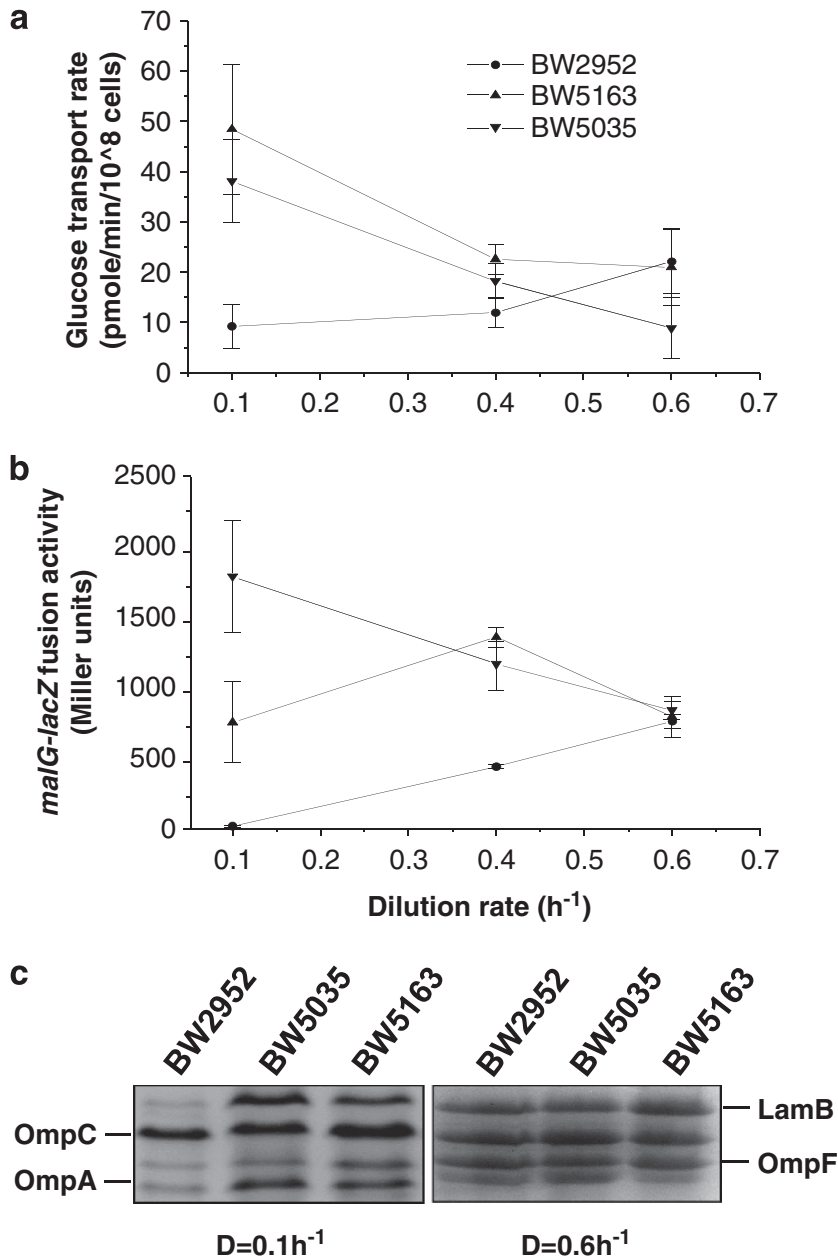

Figure 4 Changes of nutrient uptake with dilution rate in hfq mutants. Ancestor strain BW2952, $\Delta h f q$ mutant BW5035 and hfqY25D mutant BW5163 were cultured in chemostats for $40 \mathrm{~h}$ at dilution rates of $0.1 \mathrm{~h}^{-1}$, $0.4 h^{-1}$ and $0.6 h^{-1}$. Assays were then performed to determine: (a) Glucose transport rate as measured by the uptake of radiolabelled ${ }^{14} \mathrm{C}$-glucose (Notley-McRobb and Ferenci, 2000); (b) Expression of malG::lacZ gene fusion as measured by $\beta$-galactosidase activity (Notley and Ferenci, 1995). (c) Outer membrane protein profiles of the ancestor strain BW2952, $\Delta$ hfq mutant BW5035 and hfgY25D mutant BW5163 at $0.1 \mathrm{~h}^{-1}$ and $0.6 \mathrm{~h}^{-1}$ were analyzed as described in Maharjan (2006). Means and s.d.'s were obtained from 3-7 biological replicates.

fitness shift in Figure 2, so there may well be other deleterious effects of the mutations. Still, the transport advantage at $D=0.6-\mathrm{h}^{-1}$ and the viability disadvantage at $D=0.6 \mathrm{~h}^{-1}$ are likely contributing factors to the antagonistic pleiotropy as a function of growth rate.

\section{DISCUSSION}

Several alleles of $h f q$ were enriched in independent glucose-limited populations in $<100$ generations, so the parallelism is indicative of a reproducible selective advantage under nutrient limitation. Each of the alleles affected functions independently controlled by different small RNAs, such as rpoS and $p t s G$ expression, as well as porin levels (Figures 1 and 4 ). We observed no null mutations in $h f q$, but the promoter IS mutation suggests that reduced Hfq protein amount can also be a benefit at slow growth rates. An artificially constructed $h f q$ knockout had no selective advantage, explaining the absence of null 
mutants from evolving populations. This also suggests that some Hfq protein or partially functioning protein is a benefit to a cell. Given the multiplicity of $h f q$ functions, from histone-like activity to cell division and RNA degradation (Tsui et al., 1994; Gottesman, 2004), this is not altogether surprising.

The selective advantage of the various point mutations varied under the common selection condition (dilution rate $0.1 \mathrm{~h}^{-1}$ ) so there was a range of fitness effects in mutations within the same gene at the same growth rate. The Y25D substitution had the greatest mutational effect, phenotypically and in terms of benefit, followed by L46W and S60L. These amino acid residues are located in Hfq towards one face of the donut-shaped hexameric protein, as shown in Figure 6 . The substitutions occur away from the poly-U binding face and closer to the distal face shown to preferentially bind poly-A RNA and rpoS mRNA (Mikulecky et al., 2004; Link et al., 2009). We have not undertaken RNA-binding studies, but the Y25D substitution was fortuitously studied in a site-directed mutagenesis analysis, and shown to reduce rpoS mRNA binding (Mikulecky et al., 2004).

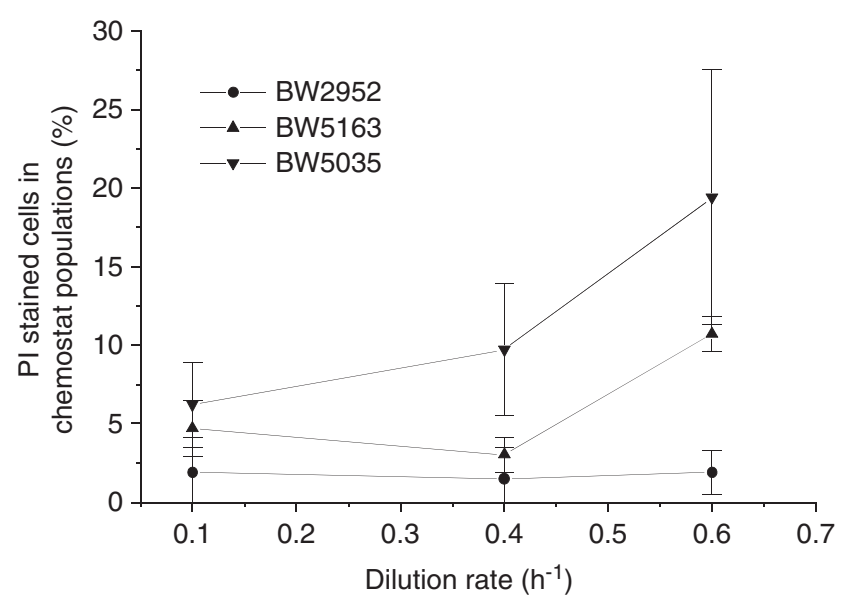

Figure 5 Changes of cell viability with dilution rate in hfq mutants. Ancestor strain BW2952, $\Delta h f q$ mutant BW5035 and hfqY25D mutant BW5163 were cultured in chemostats for $40 \mathrm{~h}$ at dilution rates of $0.1 \mathrm{~h}^{-1}$, $0.4 \mathrm{~h}^{-1}$ and $0.6 \mathrm{~h}^{-1}$. Assays were then performed to determine live- and dead-cell staining with PI. Means and s.d.'s were obtained from 3-7 biological replicates.

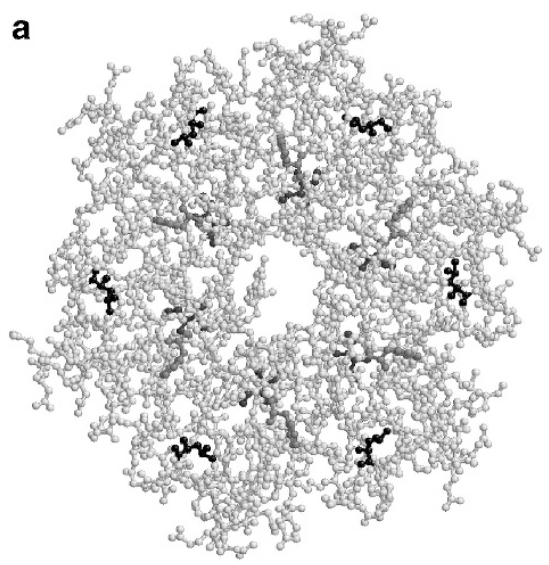

An S60A substitution was also studied (Mikulecky et al., 2004), but our S60L mutant has a greater effect on rpoS expression, probably because of the less conservative substitution involved.

The precise molecular mechanism of the negative contribution to the observed antagonistic pleiotropy present in $h f q$ mutants has not been identified so far. However, the differential effects of $h f q$ mutations on death rate at different growth rates is a likely contributor to the negative fitness at $D=0.6 \mathrm{~h}^{-1}$. One identified beneficial effect of the $h f q$ mutations is on transport, at least between dilution rates of $0.1 \mathrm{~h}^{-1}-0.4 \mathrm{~h}^{-1}$. This and other potential beneficial effects of $h f q$ mutations (Maharjan et al., 2010) are most evident at $D=0.1 \mathrm{~h}^{-1}$, the original selection condition. At high dilution rates $\left(D=0.6 \mathrm{~h}^{-1}\right)$ the transport advantage is reduced and especially between $0.4 \mathrm{~h}^{-1}$ and $0.6 \mathrm{~h}^{-1}$, a deleterious effect due to decreased viability cuts into decrease fitness. Further work is needed to identify the precise molecular mechanism of increased death rates, which appear between $D=0.1 \mathrm{~h}^{-1}$ and $D=0.6 \mathrm{~h}^{-1}$.

There are several important implications of this demonstration with $h f q$ mutants that $s$ can be extremely sensitive to slight differences in growth conditions. Firstly, as a demonstration of antagonistic pleiotropy, it is an interesting result that a mutation in $h f q$ has both beneficial and deleterious effects simultaneously, each of which varies with the growth rate. An explanation of antagonistic pleiotropy from an ecological perspective is that the growth rate is determined in chemostats by the residual glucose concentration, in other words, resource availability. So the difference in the $h f q$ mutants is between good competitive ability at $D=0.1 \mathrm{~h}^{-1}$, with low resource availability, and $D=0.6 \mathrm{~h}^{-1}$, where the residual glucose is higher in concentration (Ferenci, 2007).

A second implication of these results is for the estimation of the magnitude of $s$ in a variety of mutation analysis experiments, which generally involve competition with ancestor under a variety of conditions (Elena and Lenski, 2003; Perfeito et al., 2007; MacLean and Buckling, 2009; Lind et al., 2010McDonald et al., 2011). Fitness assays, especially in experimental evolution studies, use the selection conditions for competitions between ancestor and evolved strains. In the Lenski experiments for example, relative fitness is measured over a 24-h period in a batch culture. The bacteria experience various growth phases and growth rates in such an assay. The temporal fluctuations within a 24 -h period allow fitness to evolve in parts of the 24-h period (Rozen et al., 2009). It is not clear how many of the b

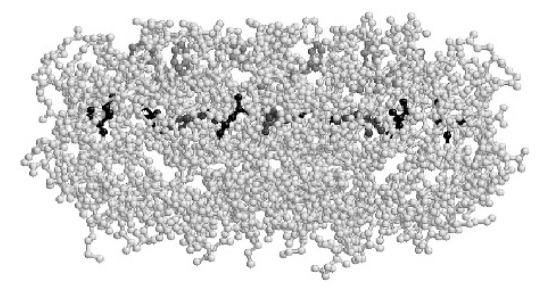

Figure 6 Structural changes in Hfq evolved in chemostat populations. Structure of the hexameric form of Hfq protein showing the sites of the residues altered in the evolved mutants. Residue Y25 is in red, L46 in black and S60 in blue, all positioned away from the distal face binding poly-A RNA and closer to the proximal face binding small-RNAs (Link et al., 2009). (a) view from the small-RNA-binding face and (b) side on. A full color version of this figure is available at the Heredity journal online. 
global regulatory mutations found in the Lenski populations (Philippe et al., 2007), or indeed in any experimental population studied by other groups, is subject to growth rate effects on fitness.

The distribution of fitness effects of mutations has been the subject of many studies (Barrett et al., 2006; Hegreness et al., 2006; Kassen and Bataillon, 2006; Perfeito et al., 2007; MacLean and Buckling, 2009; Lind et al., 2010; McDonald et al., 2011). Interestingly, the range of fitness effects for the $h f q$ alleles studied here is quite different under slightly different conditions for testing fitness at the various dilution rates. As shown by the s.d.'s at different dilution rates (Figure 3a), there are growth rates at which the different alleles have similar fitnesses but others where there is significant variation between alleles. Also, particular alleles are more prone to have variable $s$ values at different growth rates. Here again, we need to invoke differential changes in the mutants altered at different positions in Hfq and the interaction with multiple protein and RNA partners to explain this phenomenon. Although this variance may be restricted to pleiotropic regulatory mutations such as in $h f q$, it does raise a more general question as to the plasticity of fitness distributions in general.

An important reason for estimating $s$ is to predict the fixation of mutations in an evolving population. The results presented here suggest that the growth rate difference between ancestor and an evolved strain with a beneficial mutation can strongly affect the benefit over the course of fixation. In a selection environment like the chemostat, it is likely that the fixation trajectory of each $h f q$ mutation in the E. coli populations is complicated by the changing growth rate advantage of the mutants. If initially a population is growing at a $0.1 \mathrm{~h}^{-1}$ dilution rate in the selection environment, a newly mutated $h f q$ strain with better nutrient uptake has a growth rate advantage over the majority ancestral population when growing on the available residual glucose. We do not know the residual glucose concentration when the strains evolved or the growth rate when rare, but if the available glucose permits, for example, a growth rate $\mu$ of $0.2 \mathrm{~h}^{-1}$, then the mutant will have a strong positive benefit. If, however, the initial residual glucose allows a $\mu$ of $0.5 \mathrm{~h}^{-1}$, then the data in Figure 2 suggests that the advantage of the $h f q$ mutant is reduced, so is effectively subject to negative feedback with higher growth rate. If the mutants increase in proportion and the population eventually consists of a majority of evolved bacteria, these reduce residual glucose levels so $\mu$ is gradually reduced towards 0.1 (the set dilution rate). Speculatively, the non-constant benefit over the trajectory of fixation can look like frequency dependence; when in low abundance, the mutant will be subject to negative feedback because of the potential for increased growth rate, which will be relieved later at higher mutant abundance when the growth rate approaches the set dilution rate. Further study is required to establish whether the growth rate dependence of fitness can be an explanation of frequency dependence without outside ecological interactions. If so, this would be an important explanation of a wide range of phenomena where frequency dependence is either observed or postulated.

In summary, we demonstrated a series of distinct growth rate vs $s$ profiles for the alleles of the same gene, because of antagonistic effects involving the pleiotropic $h f q$ gene. Firstly, in a glucose-limited environment we find the benefit comes from improved glucose transport, but this is restricted to slow growth conditions. At faster growth rates, this benefit disappears, but an increasing defect in cell viability caused by the $h f q$ mutations results in negative fitness. The variation in $s$ demonstrated in the same environment but at different growth rates with $h f q$ mutations has relevance to $s$ estimations, description of the fitness distribution of mutations, as well as frequency-dependent behavior in the absence of obvious ecological interactions.

\section{DATA ARCHIVING}

Data deposited in the Dryad repository: doi:10.5061/dryad.5bd50.

\section{CONFLICT OF INTEREST}

The authors declare no conflict of interest.

\section{ACKNOWLEDGEMENTS}

We thank Luis-Miguel Chevin for helpful comments on an earlier version of the manuscript and the Australian Research council for funding support.

Barrett RDH, M'Gonigle LK, Otto SP (2006). The distribution of beneficial mutant effects under strong selection. Genetics 174: 2071-2079.

Barrier M, Robichaux RH, Purugganan MD (2001). Accelerated regulatory gene evolution in an adaptive radiation. Proc Natl Acad Sci USA 98: 10208-10213.

Chevin LM (2011). On measuring selection in experimental evolution. Biol Lett 7 : 210-213.

Chou HH, Berthet J, Marx CJ (2009). Fast growth increases the selective advantage of a mutation arising recurrently during evolution under metal limitation - art. no. e1000652. PLoS Genet 5: 652-652.

Chou HH, Chiu HC, Delaney NF, Segre D, Marx CJ (2011). Diminishing returns epistasis among beneficial mutations decelerates adaptation. Science 332: 1190-1192.

Conrad TM, Joyce AR, Applebee MK, Barrett CL, Xie B, Gao Y et al. (2009). Whole-genome resequencing of Escherichia coli K-12 MG1655 undergoing short-term laboratory evolution in lactate minimal media reveals flexible selection of adaptive mutations. Genome Biol 10: 10.

Cooper VS, Bennett AF, Lenski RE (2001). Evolution of thermal dependence of growth rate of Escherichia coli populations during 20,000 generations in a constant environment. Evolution 55: 889-896

Cooper VS, Lenski RE (2000). The population genetics of ecological specialization in evolving Escherichia coli populations. Nature 407: 736-739.

Death A, Notley L, Ferenci T (1993). Derepression of LamB protein facilitates outer membrane permeation of carbohydrates into Escherichia coli under conditions of nutrient stress. J Bacteriol 175: 1475-1483.

Dykhuizen DE, Hartl DE (1983). Selection in chemostats. Microbiol Rev 47: 150-168.

Elena SF, Lenski RE (2003). Evolution experiments with microorganisms: the dynamics and genetic bases of adaptation. Nat Rev Genet 4: 457-469.

Ferenci T (1996). Adaptation to life at micromolar nutrient levels: the regulation of Escherichia coli glucose transport by endoinduction and cAMP. FEMS Microbiol Rev 18: 301-317.

Ferenci T (2005). Maintaining a healthy SPANC balance through regulatory and mutational adaptation. Mol Microbio/ 57: 1-8.

Ferenci T (2007). Bacterial physiology, regulation and mutational adaptation in a chemostat environment. Adv Microb Physiol 53: 169-229.

Ferenci T, Zhou ZM, Betteridge T, Ren Y, Liu Y, Feng L et al. (2009). Genomic sequencing reveals regulatory mutations and recombinational events in the widely used MC4100 lineage of Escherichia coli K-12. J Bacteriol 191: 4025-4029.

Gottesman S (2004). The small RNA regulators of Escherichia coli: roles and mechanisms. Annu Rev Microbiol 58: 303-328.

Haldane JBS (1927). The mathematical theory of natural and artificial selection, part V: selection and mutation. Proc Camb Philos Soc 23: 838-844.

Hegreness M, Shoresh N, Hartl D, Kishony R (2006). An equivalence principle for the incorporation of favorable mutations in asexual populations. Science 311: 1615-1617.

Jörgensen F, Kurland CG (1987). Death rates of bacterial mutants. FEMS Microbiol Lett 40: 43-46.

Kassen R, Bataillon T (2006). Distribution of fitness effects among beneficial mutations before selection in experimental populations of bacteria. Nat Genet $\mathbf{3 8}$ : 484-488.

Khan AI, Dinh DM, Schneider D, Lenski RE, Cooper TF (2011). Negative epistasis between beneficial mutations in an evolving bacterial population. Science 332: 1193-1196.

King T, Seeto S, Ferenci T (2006). Genotype-by-environment interactions influencing the emergence of rpoS mutations in Eschetichia coli populations. Genetics 172 : 2071-2079.

Kishony R, Leibler S (2003). Environmental stresses can alleviate the average deleterious effect of mutations. J Biol 2: e14.

Klumpp S, Zhang ZG, Hwa T (2009). Growth rate-dependent global effects on gene expression in bacteria. Cell 139: 1366-1375.

Le Gac M, Doebeli M (2010). Epistasis and frequency dependence influence the fitness of an adaptive mutation in a diversifying lineage. Mol Ecol 19: 2430-2438.

Lind PA, Berg OG, Andersson DI (2010). Mutational robustness of ribosomal protein genes. Science 330: 825-827.

Link TM, Valentin-Hansen P, Brennan RG (2009). Structure of Escherichia coli Hfq bound to polyriboadenylate RNA. Proc Natl Acad Sci USA 106: 19286-19291.

MacLean RC, Bell G, Rainey PB (2004). The evolution of a pleiotropic fitness tradeoff in Pseudomonas fluorescens. Proc Natl Acad Sci USA 101: 8072-8077. 
MacLean RC, Buckling A (2009). The distribution of fitness effects of beneficial mutations in Pseudomonas aeruginosa - art. no. e1000406. PLoS Genet 5: 406-406.

Maharjan R, Seeto S, Notley-McRobb L, Ferenci T (2006). Clonal adaptive radiation in a constant environment. Science 313: 514-517.

Maharjan R, Zhou ZM, Ren Y, Li Y, Gaffe J, Schneider D et al. (2010). Genomic identification of a novel mutation in $h f q$ that provides multiple benefits in evolving glucose-limited populations of Escherichia coli. J Bacteriol 192: 4517-4521.

Majdalani N, Vanderpool CK, Gottesman S (2005). Bacterial small RNA regulators. Crit Rev Biochem Mol Biol 40: 93-113.

McDonald MJ, Cooper TF, Beaumont HJE, Rainey PB (2011). The distribution of fitness effects of new beneficial mutations in Pseudomonas fluorescens. Biol Lett 7: 98-100.

Mikulecky PJ, Kaw MK, Brescia CC, Takach JC, Sledjeski DD, Feig AL (2004). Escherichia coli $\mathrm{Hfq}$ has distinct interaction surfaces for DsrA, rpoS and poly(A) RNAs. Nat Struct Mol Biol 11: 1206-1214.

Miller J (1972). Experiments in Molecular Genetics. Cold Spring Harbor Laboratory: Cold Spring Harbor, NY.

Notley-McRobb L, Ferenci T (1999). Adaptive mg/-regulatory mutations and genetic diversity evolving in glucose-limited Escherichia coli populations. Environ Microbiol 1 33-43.

Notley-McRobb L, Ferenci T (2000). Substrate specificity and signal transduction pathways in the glucose-specific enzyme II (EIIGIc) component of the Escherichia coli phosphotransferase system. J Bacteriol 182: 4437-4442.

Notley-McRobb L, King T, Ferenci T (2002). rpoS mutations and loss of general stress resistance in Escherichia coli populations as a consequence of conflict between competing stress responses. J Bacteriol 184: 806-811.

Notley-McRobb L, Seeto S, Ferenci T (2003). The influence of cellular physiology on the initiation of mutational pathways in escherichia coli populations. Proc $R$ Soc Lond Ser B-Biol Sci 270: 843-848.

Notley L, Ferenci T (1995). Differential expression of mal genes under cAMP and endogenous inducer control in nutrient stressed Escherichia coli. Mol Microbiol 16 121-129.

Notley L, Ferenci T (1996). Induction of RpoS-dependent functions in glucose-limited continuous culture: what level of nutrient limitation induces the stationary phase of Escherichia coli? J Bacteriol 178: 1465-1468.
Orr HA (2009). Fitness and its role in evolutionary genetics. Nat Rev Genet 10: 531-539. Ostman B, Hintze A, Adami C (2012). Impact of epistasis and pleiotropy on evolutionary adaptation. Proc Roy Soc B-Biol Sci 279: 247-256.

Ostrowski EA, Woods RJ, Lenski RE (2008). The genetic basis of parallel and divergent phenotypic responses in evolving populations of Escherichia coli. Proc Roy Soc B-Biol Sci 275: 277-284.

Perfeito L, Fernandes L, Mota C, Gordo I (2007). Adaptive mutations in bacteria: high rate and small effects. Science 317: 813-815.

Philippe N, Crozat E, Lenski RE, Schneider D (2007). Evolution of global regulatory networks during a long-term experiment with Escherichia coli. Bioessays 29: 846-860.

Rozen DE, Philippe N, Arjan de Visser J, Lenski RE, Schneider D (2009). Death and cannibalism in a seasonal environment facilitate bacterial coexistence. Ecol Lett 12 34-44.

Scott M, Gunderson CW, Mateescu EM, Zhang ZG, Hwa T (2010). Interdependence of cel growth and gene expression: origins and consequences. Science 330: 1099-1102.

Stearns FW (2010). One hundred years of pleiotropy: a retrospective. Genetics 186 767-773.

Tsui HC, Leung HC, Winkler ME (1994). Characterization of broadly pleiotropic phenotypes caused by an hfq insertion mutation in Escherichia coli K-12. Mol Microbiol 13: 35-49.

Tweeddale H, Notley-McRobb L, Ferenci T (1998). Effect of slow growth on metabolism of Escherichia coli, as revealed by global metabolite pool ("metabolome") analysis. $J$ Bacteriol 180: 5109-5116.

Večerek B, Rajkowitsch L, Sonnleitner E, Schroeder R, Bläsi U (2008). The C-terminal domain of Escherichia coli $\mathrm{Hfq}$ is required for regulation. Nucleic Acids Res 36: 133-143.

Wang L, Spira B, Zhou ZM, Feng L, Maharjan RP, Li XM et al. (2010). Divergence involving global regulatory gene mutations in an Escherichia coli population evolving under phosphate limitation. Genome Biol Evol 2: 478-487.

Wilson DN, Nierhaus KH (2007). The weird and wonderful world of bacterial ribosome regulation. Crit Rev Biochem Mol Biol 42: 187-219.

Yu DG, Court DL (1998). A new system to place single copies of genes, sites and lacZ fusions on the Escherichia coli chromosome. Gene 223: 77-81. 